\title{
Is There a Role for Neural Crest Stem Cells in Periodontal Regeneration?
}

\author{
Atsushi Tomokiyo $^{1} \cdot$ Kim Hynes $^{2,3} \cdot$ Stan Gronthos $^{3,4}$ - Naohisa Wada ${ }^{5}$. \\ P. Mark Bartold ${ }^{2}$
}

Published online: 8 October 2015

(C) Springer International Publishing AG 2015

\begin{abstract}
The ultimate goal of periodontal treatment is not only the stabilization of periodontal disease but also regeneration of the periodontal tissues destroyed by severe periodontitis. The tissue engineering triad of stem cells, biological signals, and cell-seeded scaffold provides a biological template for tissue regeneration. Neural crest stem cells (NCSC) comprise a transient multipotent stem cell population that initially emerges from the junction of the epidermal and neural ectoderm through reciprocal interactions between these tissues and signals from the mesoderm during embryonic development of vertebrates. The cells then migrate to their destination in the embryo and differentiate into various dental mesenchymal stem cells which give rise to most components of the dental tissues, including periodontal ligament (PDL), alveolar bone, cementum, dental pulp, and dentin. The crucial role that NCSC play in embryonic development makes them a highly promising stem cell population for use in the regeneration of periodontal tissues. Here, we review the current understanding of the features and functions of NCSC-derived cells in dental
\end{abstract}

This article is part of the Topical Collection on Stem-Cell Biology for Tooth and Periodontal Regeneration

Atsushi Tomokiyo

tomokiyo@dent.kyushu-u.ac.jp

1 Department of Endodontology and Operative Dentistry, Faculty of Dental Science, Kyushu University, Fukuoka, Japan

2 Colgate Australian Clinical Dental Research Centre, School of Dentistry, University of Adelaide, Adelaide, Australia

3 South Australian Health and Medical Research Institute, Adelaide, SA, Australia

4 Mesenchymal Stem Cell Laboratory, School of Medicine, Faculty of Health Sciences, University of Adelaide, Adelaide, SA, Australia

5 Division of Oral Clinic, Kyushu University Hospital, Fukuoka, Japan tissues. We additionally discuss the possibility that NCSC contribute to the regeneration of periodontal tissues.

Keywords Neural crest $\cdot$ Tooth $\cdot$ Regeneration $\cdot$ Dental stem cells $\cdot$ Multipotency $\cdot$ Self-renewal

\section{Introduction}

Periodontitis is a chronic inflammatory condition of the periodontium induced by a polymicrobial infection [1]. In its advanced form, disease progresses with the loss of collagen fibers and attachment to the cementum surface, apical migration of the pocket epithelium, formation of deepened periodontal pockets, and resorption of alveolar bone, and finally, if left untreated, may result in tooth loss [2]. The loss of one or more teeth negatively affects individual's oral health and quality of life because it reduces a person's facial esthetics and can hamper speech and mastication. Therefore, the regeneration of the periodontal tissues destroyed by advanced periodontitis would be important to maintain teeth in good function and improve oral health-related quality of life.

Stem cells have the capacity to replicate to generate identical versions of themselves and to differentiate into multiple cell types of various tissues or organs. These properties of stem cells make them an ideally suited for use in the field of cell-based regenerative therapy [3]. Neural crest stem cells (NCSC) are a transient population of multipotent stem cells that originate from the neural crest, the interface between the non-neural ectoderm and the neural plate, during embryonic development of vertebrates. NCSC migrate into different parts of the body where they contribute to the formation of diverse cell lineages and structures, including sensory, autonomic, and enteric ganglia within the peripheral nervous system, the adrenal medulla, melanocytes, and a range of skeletal, 
connective, and endocrine cells [4]. They also have the potential to differentiate into a variety of dental mesenchymal stem cells and give rise to many dental tissues including dental pulp, apical papilla, dental follicle, and PDL [5]. The role of NCSC in tooth development has been demonstrated in chick embryos, whereby transplantation of mouse NCSC induced the generation of tooth germ structures [6].

The histological similarities between fetal development and adult tissue repair were demonstrated; at the site of skeletal injury, repair starts from the formation of a cartilage scaffold, which is gradually replaced by bone [7] and this healing process closely recapitulates the steps involved in fetal endochondral ossification. Therefore, stem cells are suggested to recapitulate developmental processes during tissue regeneration. Based on this concept, the involvement of NCSC in a wide variety of tissue development offers the potential of NCSC to be used in regenerative medicine. Recent reports have demonstrated that NCSC are present not only in the neural crest in the embryo but also in their target tissues in fetal and adult tissues; human epidermal NCSC are multipotent remnants in a postnatal location. For example, NCSC can be found in the bulge of hair follicles where they persist postnatally and into adulthood [8-10]. In addition, many reports have identified the presence of stem cells in NCSC-derived dental tissues such as dental follicle, apical papilla, pulp, exfoliated deciduous teeth, and PDL, and numerous in vitro and in vivo studies [16-22, 24-45, 49-69].

The aim of this review is to summarize the current status in NCSC biology, the potential benefits of using NCSC to treat damaged periodontal tissues, and future potential for NCSCbased regenerative periodontal therapies.

\section{Tooth Development}

The tooth germ is an aggregation of cells that eventually forms a tooth and tooth germ development proceeds through a series of morphological stages, known as the bud, cap, and bell stages [11]. A number of sequential and reciprocal interactions between the epithelial and mesenchymal tissues regulate both morphogenesis and cell differentiation through these stages [12]. The involvement of NCSC in tooth development has been studied in Wnt1-Cre/R26R transgenic mice where it has been noted that dental mesenchyme is mainly composed of NCSC-derived cells [5]; the first sign of tooth germ development is identified as a thickening of the oral epithelium, which subsequently buds into the underlying mesenchyme. At this time, NCSC-derived cells condensed around the epithelium to form the cell mass where cells change their shape and size dynamically and begin to form the tooth bud. At the cap stage, NCSC-derived cells are present in the dental papilla that plays crucial roles in epithelial-mesenchymal interactions responsible for tooth morphogenesis. In addition, these cells are also present in the dental follicle, a loose connective tissue sac that is the origin of PDL, cementum, and alveolar bone. At the bell stage, dental papilla cells directly underlying the inner dental epithelium layer induced to form odontoblasts, which differentiate and begin to synthesize dentin, and remaining dental papilla cells give rise to dental pulp. Surprisingly, NCSCderived cells also have been detected in the dental epithelial tissues including enamel organ, outer enamel epithelium, stellate reticulum, stratum intermedium, and inner enamel epithelium [13]. These results suggested that NCSC contribute to the tooth development through their potential to differentiate into a various types of cells that form dental mesenchyme and a part of dental epithelium. On the other hand, differentiated NCSC-derived cells would be lineagecommitted depending on the position of their distribution in the tooth germ.

\section{Dental Follicle Cells}

The dental follicle is a NCSC-derived ectomesenchymal structure that surrounds the enamel organ and the dental papilla of the developing tooth germ before tooth eruption [14]. When a tooth erupts, the dental follicle cells (DFC) differentiate into periodontal ligament (PDL) cells to form periodontal ligament which serves to anchor a tooth in its socket to the surrounding alveolar bone [15]. DFC near the newly forming root also differentiates into cementum forming cementoblasts. The cells towards the alveolar bone differentiate into osteoblasts responsible for the formation of bone matrix [15]. From these observations, it has been proposed that dental follicle harbors stem/progenitor cells for the periodontium [16]. When DFC were implanted with hydroxyapatite (HA) scaffolds into the dorsal surfaces of immunodeficient mice, they formed PDL-like fibrous and cementum-like mineralized tissues and expressed cementum attachment protein, bone sialoprotein (BSP), osteocalcin (OCN), osteopontin (OPN), and type I collagen (COLI) [17]. This suggests that DFC have the potential to regenerate the structures comprising the periodonium. In addition, the osteogenic potential of bone marrow-derived mesenchymal stem cells (BMMSC), skinderived mesenchymal stem cells, and DFC has been investigated using an in vivo mouse model [18]. The cells were mixed with demineralized bone matrix and fibrin gel scaffolds and transplanted into the subcutaneous tissues. Four weeks after implantation, all groups had produced new bone-like tissues with high OCN expression and radio-opacity [18]. Interestingly, the DFC-grafted group exhibited higher OCN expression and calcium content compared with BMMSC and skin-derived mesenchymal stem cells, suggesting the high potential of DFC to regenerate the mineralized tissues. 


\section{Apical Papilla Stem Cells}

The apical papilla, a soft tissue which is loosely attached to the apex of the developing tooth root, is derived from the neural crest [19]. A population of stem cells has been identified in the root apical papilla of human teeth and termed stem cells from the apical papilla (SCAP) [20]. SCAP express mesenchymal stem cells (MSC)-related cell surface markers, CD29, CD73, CD90, CD105, CD106, CD146, CD166, and STRO-1. SCAP also express periostin and $\mathrm{S} 100 \mathrm{~A} 4$, and this expression pattern is similar to that of periodontal ligament stem cells [19]. In addition, SCAP express neural crest markers including nestin, musashi-1, p75NTR, snail-1, -2, slug, and Sox9 [21]. Previous reports have demonstrated the ability of SCAP to differentiate into osteoblasts. Furthermore, SCAP have a higher population doubling capacity and produce significantly greater amounts of mineralized matrices compared to periodontal ligament stem cells (PDLSC) [22]. A bioengineered tooth root has been developed which was composed a rootshaped HA/tricalcium phosphate (TCP) scaffold loaded with SCAP and PDLSC [18]. Three months after transplantation into an extraction socket, a layer of dentin-like tissue formed on the bioengineered tooth root surface and a PDL-like tissue that had a natural relationship with the surrounding bone was generated [20]. These results demonstrated that SCAP is a highly promising cell source that has the potential to generate dentin and PDL tissues in vivo.

\section{Dental Pulp Stem Cells}

Dental pulp is a highly vascularized connective tissue in the center of the tooth that originates from neural crest [23]. Dental pulp stem cells (DPSC) were first identified in the dental pulps of human third molars [22]. DPSC have a higher proliferation rate and colony-forming ability than BMMSC [24]. DPSC were reported to express MSC-related cell surface markers, CD9, CD13, CD29, CD44, CD49a, CD49b, CD49c, CD49d, CD49e, CD51/61, CD54, CD62E, CD71, CD73, CD90, CD102, CD105, CD106, CD119, CD146, CD120a, CD166, CD271, and CD318 [25] as well as neural crest marker genes, Gsc, GATA6, TrkC, PDGFRA, p75NTR, Twist, Snail, Slug, Sox10, NCAM, and Musashi1 [26]. DPSC have the capacity to differentiate into osteoblasts as evidenced by the formation of mineralized nodules; however, the pattern of forming mineralized nodules in DPSC was different from that in BMMSC; DPSC produced only sporadic but densely mineralized nodules, whereas BMSC formed extensive sheets of mineralized deposits [24]. DPSC have also been shown to differentiate into adipocytes, chondrocytes, hepatocytes, melanocytes, and neural cells [27-30]. In addition, DPSC promoted the formation of mature blood vessels in endothelial cells by secreting VEGF [31]. The potential of DPSCs to generate the dentin-pulp complex in vivo was demonstrated; DPSCs transplanted into the dorsal surface of immunocompromised mice using HA/TCP scaffolds generated dentin-like structures on the surfaces of the scaffolds [32]. The odontoblast-like cells elongated their processes into the dentin matrix, which interacted with pulp-like interstitial tissuecontaining blood vessels. Subsequent investigations transplanted DPSCs seeded in various types of scaffolds to determine the optimal scaffold for dentin/pulp tissue engineering [33-35]. The capacity of DPSC to induce periodontal regeneration has been investigated following DPSC transplantation into periodontal defects that were surgically developed on the mesial surfaces of dog first molars [36]. The amount of newly-formed cementum and PDL formation in the DPSC transplantation groups was significantly larger than that of the control groups. In addition, a 3D root shape scaffold that was composed of a root-shaped HA/TCP scaffold containing DPSC and PDLSC was transplanted into the socket of an extracted tooth in minipigs [35]. At 6-month posttransplantation, this cell-scaffold complex induced the formation of bonelike tissue in the tooth socket. The PDL-like tissue that was inserted into bone-like tissue and positive for COLI and von Willebrand factor was also generated along a dentin-like matrix structure. Furthermore, when a pre-fabricated porcelain crown was attached to the bioengineered tooth root, this crown/root complex revealed high compressive strength. Together, these findings demonstrate that DPSC have the great capacity to generate a complete dentin-pulp-PDL complex that resists the occlusal force.

\section{Exfoliated Deciduous Teeth}

In 2003, Miura et al. were the first to report that exfoliated human deciduous teeth contained stem cell populations, which they termed stem cells from human exfoliated deciduous teeth (SHED) [37]. SHED are particularly attractive sources of stem cells because they can be isolated noninvasively from naturally exfoliated deciduous teeth. SHED have been shown to express the MSC-related cell surface markers, CD13, CD29, CD44, CD73, CD90, CD105, CD146, CD166, and STRO-1 [37-39]. SHED expressed high levels of the growth factors, basic fibroblast growth factor (bFGF), connective tissue growth factor (CTGF), transforming growth factor (TGF)- $\beta 2$, and TGF- $\beta 3$, compared with DPSC [40]. These growth factors and known to regulate the biological activities of many types of cells, and thus, may be secreted from SHED and function in an autocrine manner to stimulate their proliferation. The osteoblastic, chondrocytic, adipocytic, and neural cell differentiation potential of SHED was determined after culturing in each induction medium [19, 37, 41, 42]. SHED constitutively expressed vascular endothelial growth factor (VEGF) receptor (VEGFR)-1 and its co-receptor neuropilin- 
1, and moreover, could differentiate into endothelial cells via exogenous VEGF addition, as identified by the up-regulation of endothelium-related marker expression and the formation of capillary-like sprouts [43]. Surprisingly, a recent study demonstrated that SHED also secreted soluble proangiogenic factors that promoted the formation of capillary-like sprouts in endothelial cells [44]. These results suggested that SHED possessed the potential not only to differentiate into endothelial cells but also to induce the differentiation of endothelial cells that were located around them. When subcutaneously transplanted into immunocompromised mice, SHED showed the capacity to produce bone-like structures on the surface of ceramic bovine bone scaffolds [39]. SHED also exhibited the potential to differentiate into odontoblasts and endothelial cells and, consequently, promoted the formation of dentin and microvessels; SHED cultured in tooth root complexes that were composed of the roots of human premolars within nanofiber hydrogel or collagen scaffolds upregulated the expression of dentin-related genes in vitro [45]. After implantation of these complexes into the dorsum of immunocompromised mice, SHED-derived connective tissues occupied the full extension of the root canal. Moreover, the tooth root complexes containing SHED demonstrated new dentin and microvessel development throughout the length of the root. SHED would be a good candidate for the stem cell-based therapy; however, their effects on PDL tissue regeneration are still unclear.

\section{Periodontal Ligament Stem Cells}

The PDL is a highly specialized connective tissue that anchors the tooth root to the tooth socket bone, is derived from dental follicle, and originates from the neural crest [46]. PDL has been demonstrated to contain stem/progenitor cell populations that migrate from endosteal spaces and differentiate into critical PDL cell populations, fibroblasts, osteoblasts, and cementoblasts in response to their microenvironment [47, 48]. PDLSC were first isolated from the PDL tissue of extracted human third molar teeth [49]. PDLSC expressed MSC-related cell surface markers, CD10, CD13, CD26, CD29, CD44, CD71, CD73, CD90, CD105, CD106, CD146, CD166, CD349, STRO-1, STRO-3, and TNAP/MSCA-1 [50-52], as well as neural crest cell-related marker genes Snail, Slug, Twist, SOX9, SOX10, Nestin, p75NTR, CD49d, and Tuj1 $[53,54]$. PDLSC cultured in osteogenic medium can form mineralized nodules and demonstrate an increase in bonerelated gene expression [49] and in vivo transplantation of PDLSC into calvarial defects revealed the new bone formation within the defects [55, 56], suggesting that PDLSC can differentiate into osteoblasts. Additionally, several factors regulate the osteoblastic differentiation capacity of PDLSC. For example, cyclic tension forces enhance collagen synthesis, mineral deposit formation, and bone-related marker expression in PDLSC [57]. Furthermore, estrogen-related receptor $\alpha$ which is expressed throughout osteoblastic differentiation in PDLSC regulates their ALP activity, mineralized nodule formation, and bone-related marker expression [58]. The differentiation capacity of PDLSC into adipocytes and chondrocytes has been demonstrated by the development of Oil red O-positive lipid droplets and Safranin Opositive glycosaminoglycans, respectively [49, 59]. PDLSC can also differentiate into endothelial cells that have the potential to form capillary-like sprouts with lumens in vitro. In addition, PI3K inhibitor suppresses proliferation and endothelial cell-related marker expression in PDLSC, suggesting that PI3K activation plays a crucial role in endothelial cell differentiation of PDLSC [60]. PDLSC have the capacity, when cultured in serum-free neural induction media, to differentiate into various types of neural cells and generate free-floating neurospheres [61]. Interestingly, injection of PDLSC-derived neural cells into the hippocampus of immunesuppressed mice showed that not only did these cells survive but they migrated and integrated into the hippocampus of adult mouse brain [54], suggesting that PDLSC would be a powerful tool in the regenerative medicine for naural disease. Moreover, recent studies have demonstrated that PDLSC can generate retinal progenitors and pancreatic $\beta$-cells using three-dimensional culture methods [62].

The presence of NCSC was demonstrated not only in the embryo but also in the adult tissues [8-10], and our group investigated the presence of NCSC in the adult PDL tissue using two immortalized clonal PDL cell lines with multipotency [63]. One of these, cell line 1-11, had the capacity to differentiate into osteoblasts and adipocyte, whereas the other clone, cell line 1-17, could differentiate into osteoblasts, chondrocytes, adipocytes, and neural cell. Both cell lines strongly expressed MSC-related cell surface markers; however, their characteristic was partly different from BMMSC; cell line 1-17 and 1-11 definitely expressed PDL cell-related markers, Periostin and Scleraxis, whereas BMMSC did not express them. Interestingly, cell line 1-17 and 1-11 also revealed several different properties in addition to multipotency; cell line 1-17 strongly expressed OCT4 and Nanog mRNA, whereas their expression level was very low in cell line 1-11 [64]. Furthermore, cell line 1-17 included more p75NTR positive cells and showed stronger expression of $p 75 N T R$ mRNA than cell line 1-11. These results suggest that NSCS would be present in the adult PDL tissue and cell line 1-17 may be a good tool to clarify the roles and functions of NCSC in PDL tissue. Many studies have demonstrated the potential of PDLSC to regenerate periodontal structures using in vivo models [65]. The PDL tissue is primary composed of bundles of collagen fibrils [66] and the PDLSC in a hyaluronic acid- 
based scaffold transplanted into a subcutaneous pocket in immunocompromised mice induced a greater deal of collagen synthesis than adipose-derived stem cells [67], suggesting that PDLSC would have a greater potential to form the PDL tissue than adipose-derived stem cells. Transplantation of PDLSC into the dorsal surfaces of immunocompromised mice with HA or $\beta$-TCP scaffolds, led to the formation of various types of dental tissues, such as bone, cementum, dentin, pulp, and PDL tissues have been identified around the scaffolds [49]. PDLSC sheet combined with a dentin block scaffold has also been shown to develop cementum-like mineralized tissues and PDL-like collagen fibers [68]. In another study, fenestration defects were surgically created in nude rats, and then architectural scaffolds were designed to correspond with the defects containing perpendicular and oblique orientation of the PDL [69]. These constructs were implanted into the defects and resulted in the generation of new bone, cementum, and specific oriented fibrous bundles that were attached and anchored in a manner similar to mature, healthy periodontal complexes. Moreover, transplantation of autologous PDLSC into deep infrabony defects in three periodontitis patients resulted in significant improvement in probing depth, gingival recession, and attachment gain, which persisted for up to 72 months without immune rejection and tumorigenesis [50].

\section{Future Perspectives}

As reviewed in this article, it has been revealed that NCSC are involved in the development and regeneration of dental tissues including PDL. Our understanding of the cellular and molecular biology of NCSC-derived dental stem cells has progressed. It is now necessary to obtain a better understanding of the capacity of these cells for driving periodontal regeneration. Adult dental tissues retain populations of NCSC that show pluripotency and neural crest marker expression; however, it is extremely difficult to isolate these cells because of their low numbers in vivo. Induced pluripotent stem cells (iPSC) are amongst the most promising cell sources for tissue engineering because iPSC show high multi-differentiation and self-renewal capabilities [70, 71]. Recently, several studies have reported the successful generation of NCSC from iPSC [72-76]. Interestingly, iPSC-derived NCSC showed no tumor formation after their transplantation into nude mice and could differentiated into odontoblast-like cells that expressed odontoblast markers, DSP, Pax9, Msx1, and Lhx6 [76]. Since NCSC generated from iPSC would overcome the rarity of NCSC, suppress the risk of iPSC-related tumorigenesis, and differentiate into dental cells, it is therefore possible that these cells could become the substitute of NCSC in adult tissues and the promising cells for the realization of dental tissue engineering. Further work is now needed to confirm their safety and to evaluate their potential to regenerate dental tissues including the periodontium.

\section{Compliance with Ethical Standards}

Conflict of Interest The authors have nothing to disclose.

Human and Animal Rights and Informed Consent This article does not contain any studies with human or animal subjects performed by any of the authors.

\section{References}

1. Ellison SA. Oral bacteria and periodontal disease. J Dent Res. 1970;49(2):198-202.

2. Aida J, Ando Y, Akhter R, Aoyama H, Masui M, Morita M. Reasons for permanent tooth extractions in Japan. J Epidemiol. 2006;16(5):214-9.

3. Metcalf D. Clonal analysis of proliferation and differentiation of paired daughter cells: action of granulocyte-macrophage colonystimulating factor on granulocyte-macrophage precursors. Proc Natl Acad Sci U S A. 1980;77(9):5327-30.

4. Tonks ID, Nurcombe V, Paterson C, Zournazi A, Prather C, Mould AW, et al. Tyrosinase-Cre mice for tissue-specific gene ablation in neural crest and neuroepithelial-derived tissues. Genesis. 2003;37(3):131-8.

5. Chai Y, Jiang X, Ito Y, Bringas Jr P, Han J, Rowitch DH, et al. Fate of the mammalian cranial neural crest during tooth and mandibular morphogenesis. Development. 2000;127(8):1671-9.

6. Mitsiadis TA, Cheraud Y, Sharpe P, Fontaine-Perus J. Development of teeth in chick embryos after mouse neural crest transplantations. Proc Natl Acad Sci U S A. 2003;100(11):6541-5.

7. Hall BK, Miyake T. The membranous skeleton: the role of cell condensations in vertebrate skeletogenesis. Anat Embryol (Berl). 1992;186(2):107-24.

8. Clewes O, Narytnyk A, Gillinder KR, Loughney AD, Murdoch AP, Sieber-Blum M. Human epidermal neural crest stem cells (hEPINCSC)-characterization and directed differentiation into osteocytes and melanocytes. Stem Cell Rev. 2011;7(4):799-814.

9. Sieber-Blum M, Grim M, Hu YF, Szeder V. Pluripotent neural crest stem cells in the adult hair follicle. Dev Dyn. 2004;231(2):258-69.

10. Hu YF, Zhang ZJ, Sieber-Blum M. An epidermal neural crest stem cell (EPI-NCSC) molecular signature. Stem Cells. 2006;24(12): 2692-702.

11. Thesleff I, Vainio S, Jalkanen M. Cell-matrix interactions in tooth development. Int J Dev Biol. 1989;33(1):91-7.

12. Thesleff I. Epithelial-mesenchymal signalling regulating tooth morphogenesis. J Cell Sci. 2003;116(Pt 9):1647-8.

13. Wang SK, Komatsu Y, Mishina Y. Potential contribution of neural crest cells to dental enamel formation. Biochem Biophys Res Commun. 2011;415(1):114-9.

14. Scott JH. The development and function of the dental follicle. $\mathrm{Br}$ Dent J. 1948;85(9):193-9.

15. Palmer RM, Lumsden AG. Development of periodontal ligament and alveolar bone in homografted recombinations of enamel organs and papillary, pulpal and follicular mesenchyme in the mouse. Arch Oral Biol. 1987;32(4):281-9.

16. Lekic P, McCulloch CA. Periodontal ligament cell population: the central role of fibroblasts in creating a unique tissue. Anat Rec. 1996;245(2):327-41. 
17. Saito M, Handa K, Kiyono T, Hattori S, Yokoi T, Tsubakimoto T, et al. Immortalization of cementoblast progenitor cells with Bmi-1 and TERT. J Bone Miner Res. 2005;20(1):50-7.

18. Park BW, Kang EJ, Byun JH, Son MG, Kim HJ, Hah YS, et al. In vitro and in vivo osteogenesis of human mesenchymal stem cells derived from skin, bone marrow and dental follicle tissues. Differentiation. 2012;83(5):249-59.

19. Gosau M, Gotz W, Felthaus O, Ettl T, Jager A, Morsczeck C. Comparison of the differentiation potential of neural crest derived progenitor cells from apical papilla (dNC-PCs) and stem cells from exfoliated deciduous teeth (SHED) into mineralising cells. Arch Oral Biol. 2013;58(6):699-706.

20. Sonoyama W, Liu Y, Fang D, Yamaza T, Seo BM, Zhang C, et al. Mesenchymal stem cell-mediated functional tooth regeneration in swine. PLoS One. 2006;1:e79.

21. Abe S, Hamada K, Miura M, Yamaguchi S. Neural crest stem cell property of apical pulp cells derived from human developing tooth. Cell Biol Int. 2012;36(10):927-36.

22. Chen $\mathrm{K}$, Xiong H, Huang Y, Liu C. Comparative analysis of in vitro periodontal characteristics of stem cells from apical papilla (SCAP) and periodontal ligament stem cells (PDLSCs). Arch Oral Biol. 2013;58(8):997-1006.

23. Trubiani O, Tripodi D, Delle Fratte T, Caputi S, Di Primio R. Human dental pulp vasculogenesis evaluated by CD34 antigen expression and morphological arrangement. J Dent Res. 2003;82(9): $742-7$.

24. Gronthos S, Mankani M, Brahim J, Robey PG, Shi S. Postnatal human dental pulp stem cells (DPSCs) in vitro and in vivo. Proc Natl Acad Sci U S A. 2000;97(25):13625-30.

25. Kawashima N. Characterisation of dental pulp stem cells: a new horizon for tissue regeneration? Arch Oral Biol. 2012;57(11):143958 .

26. Janebodin $\mathrm{K}$, Horst OV, Ieronimakis $\mathrm{N}$, Balasundaram $\mathrm{G}$, Reesukumal K, Pratumvinit B, et al. Isolation and characterization of neural crest-derived stem cells from dental pulp of neonatal mice. PLoS One. 2011;6(11):e27526.

27. Harrington J, Sloan AJ, Waddington RJ. Quantification of clonal heterogeneity of mesenchymal progenitor cells in dental pulp and bone marrow. Connect Tissue Res. 2014;55 Suppl 1:62-7.

28. Paino F, Ricci G, De Rosa A, D'Aquino R, Laino L, Pirozzi G, et al. Ecto-mesenchymal stem cells from dental pulp are committed to differentiate into active melanocytes. Eur Cell Mater. 2010;20:295305.

29. Ishkitiev N, Yaegaki K, Calenic B, Nakahara T, Ishikawa H, Mitiev $\mathrm{V}$, et al. Deciduous and permanent dental pulp mesenchymal cells acquire hepatic morphologic and functional features in vitro. $\mathrm{J}$ Endod. 2010;36(3):469-74.

30. Iohara K, Zheng L, Ito M, Tomokiyo A, Matsushita K, Nakashima M. Side population cells isolated from porcine dental pulp tissue with self-renewal and multipotency for dentinogenesis, chondrogenesis, adipogenesis, and neurogenesis. Stem Cells. 2006;24(11): 2493-503.

31. Gandia C, Arminan A, Garcia-Verdugo JM, Lledo E, Ruiz A, Minana MD, et al. Human dental pulp stem cells improve left ventricular function, induce angiogenesis, and reduce infarct size in rats with acute myocardial infarction. Stem Cells. 2008;26(3): $638-45$.

32. Gronthos S, Brahim J, Li W, Fisher LW, Cherman N, Boyde A, et al. Stem cell properties of human dental pulp stem cells. J Dent Res. 2002;81(8):531-5.

33. Prescott RS, Alsanea R, Fayad MI, Johnson BR, Wenckus CS, Hao J, et al. In vivo generation of dental pulp-like tissue by using dental pulp stem cells, a collagen scaffold, and dentin matrix protein 1 after subcutaneous transplantation in mice. J Endod. 2008;34(4):421-6.

34. El-Backly RM, Massoud AG, El-Badry AM, Sherif RA, Marei MK. Regeneration of dentine/pulp-like tissue using a dental pulp stem cell/poly(lactic-co-glycolic) acid scaffold construct in New Zealand white rabbits. Aust Endod J. 2008;34(2):52-67.

35. Wei F, Song T, Ding G, Xu J, Liu Y, Liu D, et al. Functional tooth restoration by allogeneic mesenchymal stem cell-based bio-root regeneration in swine. Stem Cells Dev. 2013;22(12):1752-62.

36. Khorsand A, Eslaminejad MB, Arabsolghar M, Paknejad M, Ghaedi B, Rokn AR, et al. Autologous dental pulp stem cells in regeneration of defect created in canine periodontal tissue. J Oral Implantol. 2013;39(4):433-43.

37. Miura M, Gronthos S, Zhao M, Lu B, Fisher LW, Robey PG, et al. SHED: stem cells from human exfoliated deciduous teeth. Proc Natl Acad Sci U S A. 2003;100(10):5807-12.

38. Huang GT, Gronthos S, Shi S. Mesenchymal stem cells derived from dental tissues vs. those from other sources: their biology and role in regenerative medicine. J Dent Res. 2009;88(9):792-806.

39. Wang X, Sha XJ, Li GH, Yang FS, Ji K, Wen LY, et al. Comparative characterization of stem cells from human exfoliated deciduous teeth and dental pulp stem cells. Arch Oral Biol. 2012;57(9): 1231-40.

40. Nakamura S, Yamada Y, Katagiri W, Sugito T, Ito K, Ueda M. Stem cell proliferation pathways comparison between human exfoliated deciduous teeth and dental pulp stem cells by gene expression profile from promising dental pulp. J Endod. 2009;35(11):1536-42.

41. Koyama N, Okubo Y, Nakao K, Bessho K. Evaluation of pluripotency in human dental pulp cells. J Oral Maxillofac Surg. 2009;67(3):501-6.

42. Wang J, Wang X, Sun Z, Yang H, Shi S, Wang S. Stem cells from human-exfoliated deciduous teeth can differentiate into dopaminergic neuron-like cells. Stem Cells Dev. 2010;19(9):1375-83.

43. Sakai VT, Zhang Z, Dong Z, Neiva KG, Machado MA, Shi S, et al. SHED differentiate into functional odontoblasts and endothelium. J Dent Res. 2010;89(8):791-6.

44. Yeasmin S, Ceccarelli J, Vigen M, Carrion B, Putnam AJ, Tarle SA, et al. Stem cells derived from tooth periodontal ligament enhance functional angiogenesis by endothelial cells. Tissue Eng A. 2014;20(7-8):1188-96.

45. Rosa V, Zhang Z, Grande RH, Nor JE. Dental pulp tissue engineering in full-length human root canals. J Dent Res. 2013;92(11):970 5 .

46. Carnes DL, Maeder CL, Graves DT. Cells with osteoblastic phenotypes can be explanted from human gingiva and periodontal ligament. J Periodontol. 1997;68(7):701-7.

47. Melcher AH. On the repair potential of periodontal tissues. J Periodontol. 1976;47(5):256-60.

48. McCulloch CA, Nemeth E, Lowenberg B, Melcher AH. Paravascular cells in endosteal spaces of alveolar bone contribute to periodontal ligament cell populations. Anat Rec. 1987;219(3): 233-42.

49. Seo BM, Miura M, Gronthos S, Bartold PM, Batouli S, Brahim J, et al. Investigation of multipotent postnatal stem cells from human periodontal ligament. Lancet. 2004;364(9429):149-55.

50. Trubiani O, Zalzal SF, Paganelli R, Marchisio M, Giancola R, Pizzicannella J, et al. Expression profile of the embryonic markers nanog, OCT-4, SSEA-1, SSEA-4, and frizzled-9 receptor in human periodontal ligament mesenchymal stem cells. J Cell Physiol. 2010;225(1):123-31.

51. Tomokiyo A, Maeda H, Fujii S, Monnouchi S, Wada N, Kono K, et al. A multipotent clonal human periodontal ligament cell line with neural crest cell phenotypes promotes neurocytic differentiation, migration, and survival. J Cell Physiol. 2012;227(5):2040-50.

52. Vasandan AB, Shankar SR, Prasad P, Sowmya Jahnavi V, Bhonde RR, Jyothi Prasanna S. Functional differences in mesenchymal stromal cells from human dental pulp and periodontal ligament. J Cell Mol Med. 2014;18(2):344-54.

53. Kawanabe N, Murata S, Murakami K, Ishihara Y, Hayano S, Kurosaka $\mathrm{H}$, et al. Isolation of multipotent stem cells in human 
periodontal ligament using stage-specific embryonic antigen-4. Differentiation. 2010;79(2):74-83.

54. Bueno C, Ramirez C, Rodriguez-Lozano FJ, Tabares-Seisdedos R, Rodenas M, Moraleda JM, et al. Human adult periodontal ligamentderived cells integrate and differentiate after implantation into the adult mammalian brain. Cell Transplant. 2013;22(11):2017-28.

55. Moshaverinia A, Chen C, Xu X, Akiyama K, Ansari S, Zadeh HH, et al. Bone regeneration potential of stem cells derived from periodontal ligament or gingival tissue sources encapsulated in RGDmodified alginate scaffold. Tissue Eng A. 2014;20(3-4):611-21.

56. Park JY, Yang C, Jung IH, Lim HC, Lee JS, Jung UW, et al. Regeneration of rabbit calvarial defects using cells-implanted nano-hydroxyapatite coated silk scaffolds. Biomater Res. 2015;19:7.

57. Shen T, Qiu L, Chang H, Yang Y, Jian C, Xiong J, et al. Cyclic tension promotes osteogenic differentiation in human periodontal ligament stem cells. Int J Clin Exp Pathol. 2014;7(11):7872-80.

58. Cai C, Yuan GJ, Huang Y, Yang N, Chen X, Wen L, et al. Estrogenrelated receptor alpha is involved in the osteogenic differentiation of mesenchymal stem cells isolated from human periodontal ligaments. Int J Mol Med. 2013;31(5):1195-201.

59. Choi S, Cho TJ, Kwon SK, Lee G, Cho J. Chondrogenesis of periodontal ligament stem cells by transforming growth factorbeta 3 and bone morphogenetic protein- 6 in a normal healthy impacted third molar. Int J Oral Sci. 2013;5(1):7-13.

60. Okubo N, Ishisaki A, Iizuka T, Tamura M, Kitagawa Y. Vascular cell-like potential of undifferentiated ligament fibroblasts to construct vascular cell-specific marker-positive blood vessel structures in a PI3K activation-dependent manner. J Vasc Res. 2010;47(5): 369-83.

61. Techawattanawisal W, Nakahama K, Komaki M, Abe M, Takagi Y, Morita I. Isolation of multipotent stem cells from adult rat periodontal ligament by neurosphere-forming culture system. Biochem Biophys Res Commun. 2007;357(4):917-23.

62. Huang L, Liang J, Geng Y, Tsang WM, Yao X, Jhanji V, et al. Directing adult human periodontal ligament-derived stem cells to retinal fate. Invest Ophthalmol Vis Sci. 2013;54(6):3965-74.

63. Fujii S, Maeda H, Wada N, Kano Y, Akamine A. Establishing and characterizing human periodontal ligament fibroblasts immortalized by SV40T-antigen and hTERT gene transfer. Cell Tissue Res. 2006;324(1):117-25.

64. Maeda H, Tomokiyo A, Fujii S, Wada N, Akamine A. Promise of periodontal ligament stem cells in regeneration of periodontium. Stem Cell Res Ther. 2011;2(4):33.
65. Bright R, Hynes K, Gronthos S, Bartold PM. Periodontal ligamentderived cells for periodontal regeneration in animal models: a systematic review. J Periodontal Res. 2015;50(2):160-72.

66. Sodek J, Brunette DM, Feng J, Heersche JN, Limeback HF, Melcher AH, et al. Collagen synthesis is a major component of protein synthesis in the periodontal ligament in various species. Arch Oral Biol. 1977;22(12):647-53.

67. Jung IH, Park JC, Kim JC, Jeon DW, Choi SH, Cho KS, et al. Novel application of human periodontal ligament stem cells and watersoluble chitin for collagen tissue regeneration: in vitro and in vivo investigations. Tissue Eng A. 2012;18(5-6):643-53.

68. Ji K, Liu Y, Lu W, Yang F, Yu J, Wang X, et al. Periodontal tissue engineering with stem cells from the periodontal ligament of human retained deciduous teeth. J Periodontal Res. 2013;48(1):105-16.

69. Park CH, Rios HF, Taut AD, Padial-Molina M, Flanagan CL, Pilipchuk SP, et al. Image-based, fiber guiding scaffolds: a platform for regenerating tissue interfaces. Tissue Eng Part $\mathrm{C}$ Methods. 2014;20(7):533-42.

70. Takahashi K, Yamanaka S. Induction of pluripotent stem cells from mouse embryonic and adult fibroblast cultures by defined factors. Cell. 2006;126(4):663-76.

71. Yu J, Vodyanik MA, Smuga-Otto K, Antosiewicz-Bourget J, Frane $\mathrm{JL}$, Tian S, et al. Induced pluripotent stem cell lines derived from human somatic cells. Science. 2007;318(5858):1917-20.

72. Okawa T, Kamiya H, Himeno T, Kato J, Seino Y, Fujiya A, et al. Transplantation of neural crest-like cells derived from induced pluripotent stem cells improves diabetic polyneuropathy in mice. Cell Transplant. 2013;22(10):1767-83.

73. Liu Q, Spusta SC, Mi R, Lassiter RN, Stark MR, Hoke A, et al. Human neural crest stem cells derived from human ESCs and induced pluripotent stem cells: induction, maintenance, and differentiation into functional schwann cells. Stem Cells Transl Med. 2012;1(4):266-78.

74. Saadai P, Wang A, Nout YS, Downing TL, Lofberg K, Beattie MS, et al. Human induced pluripotent stem cell-derived neural crest stem cells integrate into the injured spinal cord in the fetal lamb model of myelomeningocele. J Pediatr Surg. 2013;48(1):158-63.

75. Xu W, Wang Y, Liu E, Sun Y, Luo Z, Xu Z, et al. Human iPSCderived neural crest stem cells promote tendon repair in a rat patellar tendon window defect model. Tissue Eng A. 2013;19(21-22): 2439-51.

76. Otsu K, Kishigami R, Oikawa-Sasaki A, Fukumoto S, Yamada A, Fujiwara N, et al. Differentiation of induced pluripotent stem cells into dental mesenchymal cells. Stem Cells Dev. 2012;21(7):115664 . 